\title{
José Ballivián y la Comisión Topográfica: la institucionalización de la cartografía oficial en Bolivia (1842-1847)
}

José Ballivián et la Commission topographique

l'institutionnalisation de la cartographie officielle en Bolivie (1842-1847) José Ballivián and the Topographic Commission: the institutionalization of official cartography in Bolivia (1842-1847)

\section{Víctor Hugo Machaca Mamani}

\section{(2) OpenEdition}

\section{Edición electrónica}

URL: https://journals.openedition.org/bifea/72345

DOI: 10.4000/bifea. 12345

ISSN: 2076-5827

Editor

Institut Français d'Études Andines

\section{Edición impresa}

Fecha de publicación: 8 diciembre 2020

Paginación: 277-295

ISSN: 0303-7495

\section{REFERENCIA ELECTRÓNICA}

Víctor Hugo Machaca Mamani, «José Ballivián y la Comisión Topográfica: la institucionalización de la cartografía oficial en Bolivia (1842-1847)», Bulletin de l'Institut français d'études andines [En línea], 49 (2) / 2020, Publicado el 25 noviembre 2021, consultado el 01 diciembre 2021. URL: http://journals.openedition.org/bifea/12345 ; DOI: https://doi.org/10.4000/bifea.12345

\section{(c) $(9) \odot$}

Les contenus du Bulletin de l'Institut français d'études andines sont mis à disposition selon les termes de la licence Creative Commons Attribution - Pas d'Utilisation Commerciale - Pas de Modification 4.0 International. 


\title{
José Ballivián y la Comisión Topográfica: la institucionalización de la cartografía oficial en Bolivia (1842-1847)*
}

\author{
Víctor Hugo Machaca Mamani**
}

\begin{abstract}
Resumen
En esta investigación presentamos la Comisión Topográfica (1843-1847) como una entidad creada durante el gobierno de José Ballivián (1841-1847), cuya repercusión fue fundamentar el ideario nacional y de su territorio desde la confección de mapas geográficos. Como resultado del trabajo científico de sus miembros, se elaborarían los dos primeros mapas oficiales de Bolivia (1843 y 1859), realizados por el ingeniero francés Felipe Bertrés, el primero, y por los miembros de la Comisión Topográfica, el segundo, dirigos por los oficiales bolivianos Juan Ondarza y Juan Mariano Mujía. El accionar político de Ballivián sería determinante al momento de institucionalizar la cartografía de Estado en Bolivia.
\end{abstract}

Palabras clave: Comisión Topográfica, mapa oficial, siglo XIX, Bolivia, nación, Estado

\section{José Ballivián et la Commission topographique : I'institutionnalisation de la cartographie officielle en Bolivie (1842-1847)}

\section{Résumé}

Dans cette recherche, nous présentons la Commission topographique de Bolivie (1843-1847) comme une entité créée par le gouvernement de José Ballivián (1841-1847), qui a permis de fonder l'idéologie nationale et son territoire à partir de l'élaboration des cartes géographiques. À la suite du travail scientifique de ses membres, ont été élaborées les deux premières cartes officielles de ce pays (1843 et 1859). La première fut préparée par l'ingénieur français Philippe Bertrés et la seconde par les membres de la Commission topographique dirigée par les officiers boliviens Juan Ondarza et Juan

* Este artículo es producto de una investigación más amplia financiada gracias a la Beca Andina de Apoyo a la Investigación IFEA-UMIFRE 17 MEAE/CNRS USR 3337 América Latina del Instituto Francés de Estudios Andinos, 2018.

** Licenciado en Historia, Universidad Mayor de San Andrés. E-Mail: vicmacmam@alum.us.es 
Mariano Mujía. Les actions politiques de Ballivián furent déterminantes lors de l'établissement de la cartographie étatique en Bolivie.

Mots-clés : Commission topographique, carte officielle, 19e siècle, Bolivie, Nation, État

\title{
José Ballivián and the Topographic Commission: the institutionalization of official cartography in Bolivia (1842-1847)
}

\begin{abstract}
In this research we present the Topographic Commission of Bolivia (1843-1847) as an entity created during the government of José Ballivián (1841-1847), whose impact was to support the national and territorial ideology through the preparation of geographic maps. As a result of the scientific work of its members, the first two official maps of this country were produced in 1843 and 1859 . The first one was created by the French engineer Felipe Bertrés, and the second one by the members of Topographic Commision, under the command of the Bolivian officers Juan Ondarza and Juan Mariano Mujía. The political actions of Ballivián were decisive in establishing the state cartography in Bolivia.
\end{abstract}

Keywords: Topographic Commission, official map, 19th century, Bolivia, nation, state building

\section{INTRODUCCIÓN}

Una labor central para la consolidación de una nación es el conocimiento y control del territorio, y para ello los mapas geográficos se vuelven instrumentos básicos. En Bolivia, uno de los proyectos emprendidos por el gobierno de José Ballivián Segurola1 (1841-1847) fue el levantamiento de los primeros dos mapas oficiales del país, labor que encargó a los miembros de la Comisión Topográfica creada en 1843. Al año siguiente de facultar al ingeniero francés Felipe Bertrés 2 la elaboración de la primera carta geográfica de Bolivia en 1842, Ballivián dejó la exploración del país en manos de los oficiales Juan Ondarza3 , Juan Mariano Mujía ${ }^{4}$ y Lucio Camacho5, labor que concluyó con la publicación del segundo mapa oficial del país. Los resultados de tales trabajos debían ser las descripciones

1 Ballivián nació el 5 de mayo de 1805. Con amplia carrera militar, gobernó Bolivia entre 1841 y 1847. Falleció en Río de Janeiro en 1852 (Aranzáes, 1915: 96-104).

2 Bertrés nació en el cantón Castalnau-Magnoac al Sur de Francia al finalizar el siglo XVIII (21786?). Estudió Matemáticas e Ingeniería en la Escuela Politécnica de París, transformada posteriormente en la Escuela Superior Técnica del Ejército (1804). Llegó a Bolivia durante el gobierno del Mariscal Andrés de Santa Cruz. Desde la década de 1830 ejerció varios cargos como ingeniero hasta que, en 1844, volvió a Tucumán (Yaben, 1938: 538); falleció finalmente en la ciudad de Salta en 1856.

3 Ondarza nació en la ciudad de Sucre el 17 de mayo de 1827 (Ballivián \& Idiáquez, 1890: V). Ocupó varios cargos políticos y militares y falleció en La Paz en enero de 1874 o 1875.

4 Mujía nació en la ciudad de Sucre. Incorporado como cadete en octubre de 1841, participó en la batalla de Ingavi y, desde allí, fue escalando grados hasta llegar al de general de brigada en 1870 (Díaz Arguedas, 1929: 557).

5 El presbítero Nicanor Aranzáes indica que Camacho nació en La Paz el 7 de enero de 1832, donde ingresó a la Escuela de Arquitectura Civil y Militar que comandaba Bertrés, destacándose entre sus habilidades las relacionadas al dibujo y la pintura. Falleció en 1879 (Aranzáes, 1915: 170-171). 
detalladas de los sitios recorridos, pero sobre todo cartas topográficas que serían el sustento de un mapa nacional con precisa información geográfica recogida in situ. ¿Cómo fue creada la Comisión Topográfica? ¿Qué rol jugó en la institucionalización de la cartografía oficial en Bolivia a mediados del siglo XIX? Para ello indagaremos en las circunstancias de la elaboración del primer y segundo mapas nacionales de Bolivia en el siglo XIX, a partir del establecimiento de la Comisión Topográfica en el gobierno de José Ballivián. Consideramos esta creación como preludio de un proceso de institucionalización de la ciencia geográfica por parte del Estado, en un periodo de compleja dificultad para la producción cartográfica, ya sea por la carencia de vías de comunicación, el amplio espacio geográfico del país, la merma económica o las limitaciones propias de los miembros de la Comisión. Así, estudiaremos la historia cartográfica boliviana en relación con las políticas del gobierno de José Ballivián.

En este artículo buscamos comprender de mejor manera las políticas de José Ballivián en el decenio de 1840, complementando los trabajos de Janet Groff Greever y Pilar García Jordán, cuyos textos, José Ballivián y el oriente boliviano (1987) y Cruz y arado, fusiles y discursos. La construcción de los Orientes en el Perú y Bolivia, 1820-1940 (2001) respectivamente, se han convertido en clásicos de la historiografía para ese periodo. Igualmente, este artículo permite entender mejor cómo se fue conociendo, imaginando y representando el territorio. Consideramos que los años de gobierno de José Ballivián, y específicamente los de la Comisión Topográfica que vemos en esta investigación (1842-1847), anticiparon una etapa de institucionalización de la ciencia geográfica, la misma que se reflejó en representaciones cartográficas y políticas empleadas para el conocimiento de la geografía del país.

Para esta investigación consultamos repositorios del país, con énfasis en correspondencia del Fondo del Ministerio de Guerra del Archivo y Biblioteca Nacionales de Bolivia. Sin embargo, el volumen de documentos consultados es pequeño en relación con todo lo que suponemos produjo documentalmente el trabajo de la Comisión Topográfica, y que aún no ha sido ubicado. En el fondo mencionado, por ejemplo, no encontramos ninguna descripción o diario de campo de sus artífices. Otra información complementaria puede encontrarse en periódicos de la época, folletos y algunos documentos manuscritos; que dan cuenta, así sea parcialmente, de la labor de los cartógrafos.

De esta manera, describimos parte del periodo de gobierno de José Ballivián y su relación con el desarrollo de la ciencia geográfica, primero en el aspecto educativo y después en el plano cartográfico. En estos puntos Bertrés jugó un rol determinante. En una segunda y tercera parte, describimos las dos fases que, a nuestro criterio, implicó el trabajo de la Comisión Topográfica: su conformación y más adelante su afianzamiento, que tendría en medio un momento de transición que es importante mencionar. Este proceso asentaría las bases institucionales de la cartografía oficial en el siglo XIX en Bolivia. 


\section{JOSÉ BALLIVIÁN, FELIPE BERTRÉS Y EL DESARROLLO DE LA CIENCIA}

La historia de la ciencia ha permitido abordar diferentes aspectos de las prácticas científicas y de su razonamiento en distintas partes del mundo. Como expresa el historiador mexicano Juan José Saldaña, la historia de la ciencia muestra cómo se han constituido

... la cultura científica, las comunidades, el ethos científico particular, las escuelas de pensamiento, los mecanismos sociales de valoración del trabajo científico, las instituciones, las políticas de fomento, los establecimientos de enseñanza... (Saldaña, 2006: 16).

Superando la visión eurocéntrica del desarrollo de este saber, literatura reciente ha investigado el lugar de la ciencia latinoamericana en el siglo XIX y la repercusión de sus prácticas en el periodo de construcción nacional.

En este aspecto es interesante subrayar el rol del Estado como un «agente promotor de la ciencia» (Lafuente \& López-Ocón, 1998: 9) que ha tenido influencia e intervención al momento de conocer la naturaleza, por ejemplo, o hacer emprendimientos en aspectos tecnológicos o de industria en aquel periodo. Los gobernantes en la región pensaron y ejecutaron programas de «conocimiento y evaluación de los recursos naturales y humanos de las nuevas entidades políticas» (Lafuente \& López-Ocón, 1998: 9). Así también impulsaron proyectos para el conocimiento del territorio que se afianzaron en instituciones estatales.

En esta línea escribe Juan José Saldaña, quien destaca la noción de «comunidad científica», utilizada para comprender el rol desempeñado por el Estado y sus políticas al momento de crear y fomentar las instituciones necesarias para la extensión de la ciencia (Saldaña, 2006: 12-13). En Bolivia, el Estado, sujeto a sus posibilidades, tuvo que apoyarse en el ejército para delegar funciones topográficas, y ese factor es clave al momento de entender cómo se fue construyendo la ciencia de los mapas oficiales. Tal es el caso de la Comisión Topográfica boliviana, para lo cual se apeló a ingenieros militares.

Los primeros años del gobierno de Ballivián (1841-1843) fueron decisivos para erigir un aparato administrativo acorde a los proyectos pretendidos. Él mismo recuerda:

Desde el día memorable en que nuestras armas triunfaron en Ingavi6, me ocupé de la reorganización del país. Los decretos de esa época os acreditan mi consagración á este objeto (Ballivián, 1843: 10).

La base institucional lograría el control en los asuntos públicos del país sumado al conocimiento del territorio, proyecto emprendido a partir del fomento a las exploraciones. Pilar García Jordán menciona incluso que Ballivián «fue el primer

6 «Ingavi» hace referencia a la batalla con el mismo nombre donde se enfrentaron los ejércitos boliviano y peruano el 18 de noviembre de 1841 en las inmediaciones del municipio de Viacha (departamento de La Paz, Bolivia). 
político boliviano que planteó un plan integral para el conocimiento y control del territorio nacional» (García Jordán, 2001: 252). De esta manera, el proyecto estatal incluía a la ciencia para recorrer, conocer y graficar el territorio.

En el ámbito de la instrucción pública, José Ballivián tenía la idea de crear una institución dedicada a la enseñanza de las ciencias. Así, durante su gobierno se creó la Escuela de Arquitectura Civil y Militar como parte del Colegio de Ciencias de San Simón de La Paz el 4 de julio de 1842, conforme al reglamento del 26 de febrero de 1842. Se encomendó la dirección y organización de esta escuela al ingeniero francés Felipe Bertrés y a José María Núñez del Prado7, considerado el primer arquitecto boliviano (La Gaceta del Gobierno, 09.VII.1842).

Posteriormente impulsó el Decreto orgánico de universidades (25.VIII.1845), mediante el cual se añadieron los estudios de física y matemáticas a los anteriores de teología, derecho y filosofía (Barragan et al., 2015: 129). El Colegio de Ciencias, en el que estaba la Escuela de Arquitectura Civil y Militar, funcionó hasta que, por el Estatuto del 15 de octubre de 1845, se ordenó la reinstalación de la Universidad Mayor de San Andrés, la misma que fue llevada a cabo en acto solemne el 18 de noviembre de 1845, fecha conmemorativa por los cuatro años de la victoria en la Batalla de Ingavi. Al dejar de funcionar el Colegio de Ciencias, se creó la Facultad de Ciencias Físicas y Matemáticas en la universidad que estaba conformada por tres profesores: uno de matemáticas elevadas, otro de física y química y finalmente uno de historia natural, como figura en el Código de Instrucción Pública de 1845 (citado en Salinas, 1967: 296).

Además de esta contribución al conocimiento científico de la época, el trabajo de Bertrés en el país se desarrolló en construcciones de tipo «triunfalista», civil, religioso y militar. Otra de sus facetas fue la de cartógrafo, por la que, cuando se encontraba en Argentina, diseñó los planos de las ciudades de Tucumán y Buenos Aires. El rol que tendría Bertrés en adelante sería determinante para Ballivián, ya que proyectaría el Mapa Corográfico de la República de Bolivia. Personas como él fueron incursionando en la ciencia de la época, con la elaboración de diversos instrumentos que favorecerían el conocimiento del país y, en lo posterior, comenzarían a dibujar su geografía.

\section{PRIMERA FASE: EL MAPA DE BERTRÉS Y LA CONFORMACIÓN DE LA COMISIÓN TOPOGRÁFICA}

Además de su empeño por fomentar la instrucción en áreas como la matemática y la física, Ballivián creó organismos estatales capaces de recopilar información suficiente para el mejor conocimiento de la población y del territorio y que aporten con datos, topográficos o estadísticos. Apoyado en ingenieros militares que, en ese momento en Bolivia como en otras latitudes, eran los comisionados de la

Núñez del Prado nació en La Paz a principios del siglo XIX. Trabajó en el diseño del Teatro Municipal de La Paz, el Palacio de Gobierno y en las obras de construcción de la Catedral de La Paz (Barnadas, T. II, 2002: 653). 
cartografía estatal, decidió impulsar la elaboración de los primeros mapas sobre la base de una instancia oficial, la Comisión Topográfica, que sería la delegada de explorar los territorios y brindar datos de los mismos, que fueran útiles para lograr una mejor administración del Estado.

A dos meses de firmada la paz con Perú en Puno (1842) tras el conflicto bélico en Ingavi, el gobierno comisionó a un ingeniero francés para levantar un mapa geográfico nacional. Por orden de José Ballivián, el ministro de Guerra, general Eusebio Guilarte, envió una nota a Felipe Bertrés para que realice una carta geográfica de la república subdividida en siete departamentos. En respuesta, Bertrés indicó a Guilarte que se encontraba realizando una escala de grados para la formación de este mapa y que era de su necesidad contar con algunas descripciones y mapas para complementar su trabajo8. Siendo elaborado en poco más de un año, fue titulado como Mapa Corográfico de la República de Bolivia (1843) (fig. 1).

Pero el levantamiento del mapa fue una etapa preliminar a la creación de la Mesa Topográfica. En julio de 1843, el trabajo de Bertrés se formalizó con el establecimiento de esta institución:

\section{ORDEN GENERAL DE 8 DE JULIO}

Mesa topográfica en el E. M. G. del Ejército.

En Sucre á 8 de julio de 1843.

Art. Único. Con arreglo al artículo 61 de capítulo 1. ${ }^{\circ}$ del código militar, se establece en el E. M. G. [Estado Mayor General] la mesa topográfica, que será dirijida por el Coronel graduado Felipe Beltres [Bertrés] á quien se le dará de alta con esta fecha. Este Jefe propondrá en adelante los oficiales adjuntos y adictos, que crea necesarios para el buen desempeño de su mesa. El General Jefe.- Sagárnaga (Colección..., T. VIII, 1843: 222).

Esta orden fue asumida directamente desde el Estado Mayor General, seguramente a pedido de Ballivián. Al revisar los documentos parlamentarios de la época como los Redactores del Congreso Nacional, no encontramos mayor información sobre esta Mesa; aparecen algunas menciones en las memorias ministeriales, aunque muy escuetas. Solo Ballivián hace un comentario acerca de la creación del «Departamento Topográfico» en su Mensaje a la Convención Nacional de 1843:

Se ha creado un Departamento Topográfico, y mandado levantar mapas prolijos y ecsactos de toda la República, ofreciendo premios á los que con sus conocimientos, contribuyan à tan importante trabajo (Ballivián, 1843: 15).

El Mensaje de Ballivián está fechado en 23 de abril de 1843 cuando la Mesa Topográfica aún no había sido creada (08.07.1843). En ese momento el único mapa que se estaba elaborando era el de Felipe Bertrés pero ya anticipaba el refuerzo institucional en aras del conocimiento geográfico. 


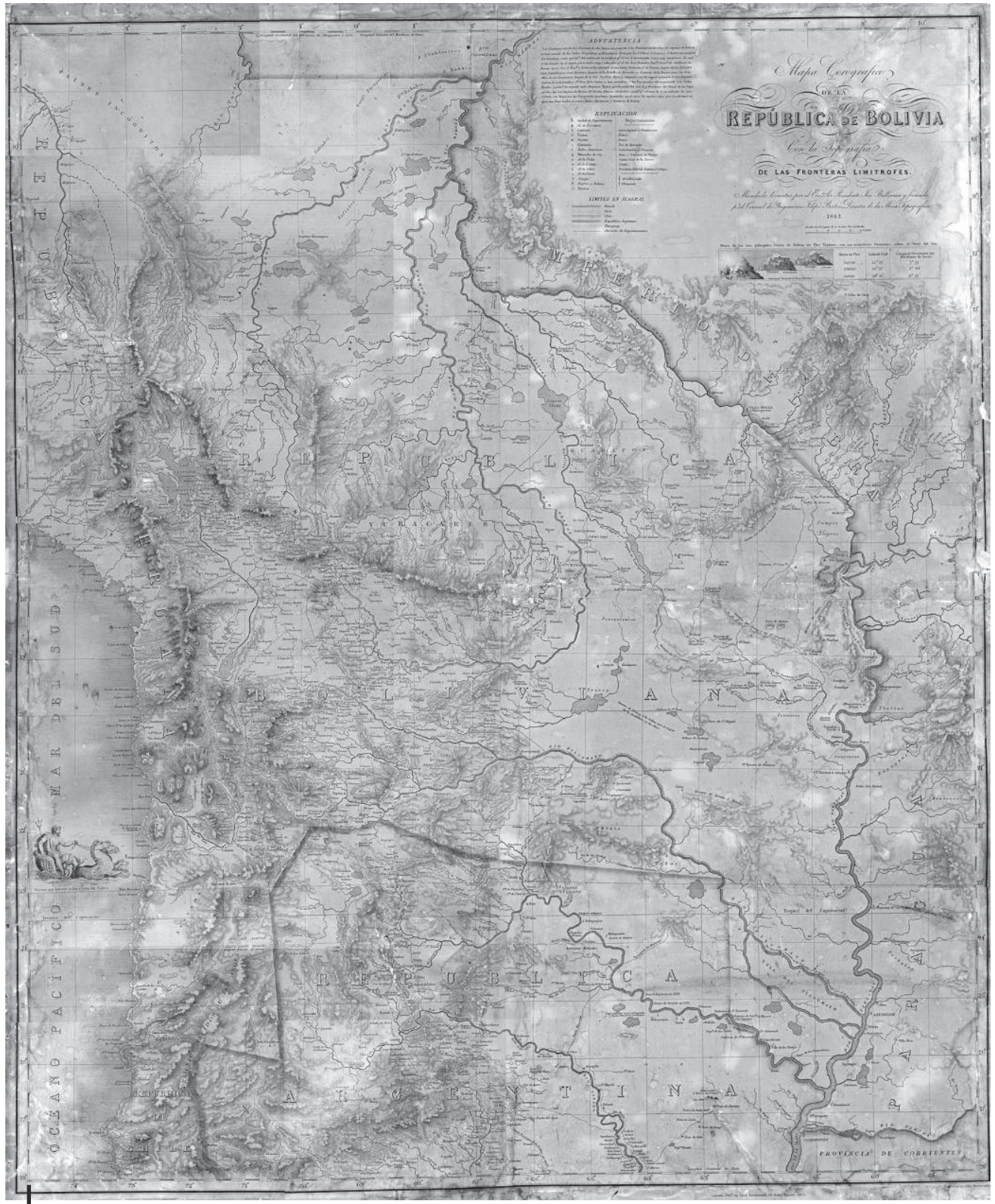

Figura 1 - Mapa Corográfico de la República de Bolivia, Felipe Bertrés, 1843

Mapa Corográfico de la República de Bolivia, mandado a levantar por el presidente José Ballivián y formado por el coronel de ingenieros Felipe Bertrés (dimensiones 126 × $105 \mathrm{~cm}$ )

Fuente: AHCDL, Bo 932, Casa de La Libertad, Sucre 
La «Mesa» se conformó de a poco. Tuvo sede, primero, en la ciudad de Sucre. A principios de 1843 y hasta el mes de mayo, Juan Ondarza figuró como cadete del Estado Mayor en esa ciudad9; ascendió el 31 de mayo a subteniente y desde el mes de junio aparece en los documentos con ese grado10. Felipe Bertrés fue incluido en las listas del Estado Mayor General del ejército —al que estuvo destinado tras su designación como jefe de la Mesa Topográfica - el 1 de agosto de 1843 en calidad de teniente coronel y con un abundante haber11. Entonces, desde agosto de aquel año Bertrés tuvo contacto con Ondarza en la ciudad de Sucre.

Más adelante, en un documento del mes de septiembre de 1843 en el que figura el sueldo recibido por los oficiales el mes anterior, el subteniente Juan Mariano Mujía ingresó en la sección del Estado Mayor General en Sucre12. En diciembre se incluyó a la misma instancia al comandante graduado Ladislao Marín13. Finalmente, en un documento para la revista de la mencionada sección de ese mes de diciembre aparecen solamente seis oficiales, entre los que resaltamos a Felipe Bertrés, Ladislao Marín, Juan Mariano Mujía y Juan Ondarza14. De esta manera, algunos de los que formarían parte de la Comisión Topográfica y sus subcomisiones se encontraban en el Estado Mayor en Sucre a finales de 1843.

Esta segunda mitad del año 1843 es determinante para la conformación de la Comisión Topográfica. Como indicaba la Orden General del 8 de julio de 1843, Bertrés podía proponer los miembros adjuntos para el desempeño de su Mesa y, mientras él culminaba su mapa entre agosto y septiembre de 1843, seguramente decidió apoyarse en algunos oficiales que eran parte del Estado Mayor en Sucre. De esta manera incluyó a Ondarza y Mujía en la Mesa Topográfica y propuso a Ladislao Marín para realizar levantamientos topográficos en el Oriente del país. Poco tiempo pasaría para que el gobierno encargara a estos últimos recorrer el país.

Ahora bien, mientras Bertrés elaboraba el «Mapa Corográfico» (en las ciudades de La Paz y Sucre), tenía contacto casi directo con Ballivián. En ocasiones se comunicaba a través de sus ministros, pero la cercanía entre el mandatario y

9 ABNB, MG, 1843, 5. Sucre, 7 de febrero de 1843; ABNB, MG, 1843, 5. Sucre, 10 de marzo de 1843; ABNB, MG, 1843, 5. Sucre, 9 de abril de 1843; ABNB, MG, 1843, 5. Sucre, 2 de mayo de 1843. En estos meses recibe un haber de doce pesos.

10 ABNB, MG, 1843, 5. Sucre, 1 de junio de 1843. Su haber era de 50 pesos y un líquido de 45. En una transcripción que hace Arturo Costa de la Torre de la hoja de servicios de Ondarza, este aparece como cadete desde junio de 1841 y con el grado de subteniente para la batalla de Ingavi en noviembre (Costa, 1970: 71-73). Según nuestra revisión de la lista de oficiales para la revista mensual y los presupuestos mensuales de los mismos, consultados en el Fondo del Ministerio de Guerra del ABNB, Juan Ondarza fue cadete del Estado Mayor hasta el mes de mayo de 1843. Recién desde junio del mismo año figura como subteniente.

11 El haber de Bertrés ascendía a 180 pesos y un líquido de 144, solo por debajo del oficial mejor pagado en esa instancia en Sucre. Podemos derivar que recibió esta suma por tratarse de un oficial extranjero y encomendado por el propio presidente Ballivián. ABNB, MG, 1843, 5. Sucre, 1 de agosto de 1843.

12 ABNB, MG, 1843, 5. Sucre, 2 de septiembre de 1843.

13 ABNB, MG, 1843, 5. Sucre, 1 de diciembre de 1843.

14 ABNB, MG, 1843, 5. Lista para la revista de la sección del Estado Mayor General. Sucre, 1 de diciembre de 1843. 
Bertrés fue notoria. Después de la finalización del mapa, Bertrés fue alejándose de la Mesa Topográfica y del país. Presumimos que su partida se debió a su salud deteriorada, como detalló su hijo Pedro Bertrés15.

\section{DE LA MESA A LA COMISIÓN, DE BERTRÉS A ONDARZA Y MUJÍA}

Desde 1844, y hasta la publicación del Mapa de la República de Bolivia en 1859, la Mesa Topográfica pasó a denominarse Comisión Topográfica16 y quienes firmaban a nombre de ella eran los oficiales Juan Ondarza y Juan Mariano Mujía17. Creemos que Juan Ondarza y Juan Mariano Mujía apoyaron a Bertrés en la elaboración del «Mapa Corográfico», recolectando los últimos datos o ayudando a representarlo en la segunda mitad de 1843, mientras aprendían el trabajo topográfico. Al finalizar este año, el gobierno ordenó a Ondarza y Mujía recorrer personalmente algunos puntos del país, como expresa propiamente Bertrés:

Hallándose casi concluidas por los Oficiales de este Cuerpo las copias parciales en escala doble de los tres Departamentos Chuquisaca, la Paz y Potosí, con parte de los colindantes á estos, y como de orden de S.E. dichos Oficiales [¿Ondarza y Mujía?] deben ir á hacer las correcciones que sean precisas en las Topografias de los referidos Departamentos ${ }^{18}$.

Ondarza expresó también haber realizado junto a Mujía «... pequeñas excursiones á 10 y 20 leguas alrededor de la Capital [Sucre] siempre bajo la dirección del Coronel Bertrés...»(Ondarza, 1911: 597), hecho que indica que el trabajo entre el francés y los bolivianos era conjunto. Se infiere que Bertrés preparaba a los miembros de la Comisión Topográfica que le suplirían en las labores de levantamiento de datos para la elaboración de mapas.

La información recogida desde entonces fue útil para proyectar el segundo mapa oficial. Al respecto, Ondarza en su documento «Autógrafo»19 detalló:

15 Mientras Pedro Bertrés solicita su licencia del ejército, menciona: «... es un dever indispensable en mí acompañarlo, como buen hijo, en su suerte y enfermedades». ABNB, MG, 20a, Carta de Pedro Bertrés. Esta carta no lleva fecha específica, pero el sello oficial corresponde al año 1844, de manera que tomamos esta referencia como la de elaboración de la misiva. De igual modo asumimos que iba dirigida al ministro del ramo de Guerra, Pedro Buitrago.

16 La denominación de «Comisión Topográfica» es la más recurrente antes de la publicación del Mapa de la República de Bolivia de 1859. Mientras la Mesa funcionaba con Bertrés, este firmó a nombre del «Departamento de Ingenieros», del «Departamento Topográfico» y del «Departamento de Topografía». Pensemos que solo al principio Bertrés realizaba el trabajo de la Mesa. Con Ondarza y Mujía se fue consolidando esta institución topográfica.

17 En un documento que se encuentra en el ABNB fechado en 1 de agosto de 1843, cuando Felipe Bertrés estaba cerca de terminar el «Mapa Corográfico», Juan Mariano Mujía todavía figura como parte del «Cuerpo de Ingenieros», con su «haver» líquido y un criado. ABNB, MG, 1843, 61. Sucre, 1 de agosto de 1843. Seguramente Mujía y Ondarza apoyaban a Bertrés en la elaboración del mapa, pero una vez que Bertrés lo terminó, tanto Mujía como Ondarza formaron parte de la Comisión Topográfica de manera formal. Así aparecen en los registros desde 1844.

18 ABNB, MG, 1843, 20b. Carta de Felipe Bertrés al ministro de Guerra. Sucre, 12 de noviembre de 1843.

19 Obtuvimos la referencia del «Autógrafo del Señor Ondarza» por medio de la obra Hombres célebres de Bolivia, Juan Ondarza, Abdón Senen Ondarza de Arturo Costa de la Torre, quien transcribió el 
En el año 1842, el Supremo Gobierno, en conformidad á muchas leyes y disposiciones preexistentes, tuvo á bien ordenar la formación de una Mesa de Topografía, anexa al Estado Mayor del Ejército. Esta mesa presidida por el Coronel Felipe Bertrés, y á [la] que fuimos incorporados, debía ocuparse principalmente de la formación de un Mapa General Geográfico de la Republica.- Se procedió desde luego á delinear uno.- Antiguas é incorrectas Topografías de algunas Provincias, varios mapas extranjeros y unos pocos libros de viajeros, he ahí el total de los elementos que se tuvieron al frente para pintar el Mapa - Corográfico, que en verdad nada ecsacto contenía, como no sean los poquísimos lugares visitados en Chuquisaca y Santa Cruz por el Coronel Bertrés, Viejo y Sabio Ingeniero.- A fuerza de desvelos, de empeño y de constancia, y luchando con la eterogeneidad de los elementos que hemos mencionado, absurdos y contradictorios en su mayor parte, es como se consiguió pintar el Mapa de Bolivia.

El Gobierno sin embargo que harto conocía los defectos de este Mapa, lo mandó publicar como un primer bosquejo que, sirviese de norma á los trabajos de la comisión estadística, creada así mismo en aquel año, y también al levantamiento de un Nuevo Mapa que debía formarse en virtud de estudios más especiales (Ondarza, 1911: 596-597)20.

Aunque Ondarza menciona que el Mapa de la República de Bolivia se elaboró desde 1842, la Mesa o Comisión Topográfica comenzó su trabajo en la segunda mitad de 1843 y al año siguiente, 1844, inició el relevamiento topográfico. Pero más allá, esta cita refleja que los dos mapas oficiales de Bolivia eran parte de un mismo proyecto geográfico, el mismo que tuvo un periodo de transición en el cual la Mesa pasó a denominarse en definitiva Comisión Topográfica y su jefatura cambió de Bertrés a Ondarza y Mujía.

De manera análoga, el Mapa Corográfico de la República de Bolivia fue un bosquejo del Mapa de la República de Bolivia y, sin dejar de ser por ello un mapa oficial, debía apoyar a la Comisión Estadística que Ballivián pretendía formar. Llama la

documento, aunque incompleto (Costa de la Torre, 1970: 88-91). De nuestra parte consultamos el Boletín de la Oficina Nacional de Estadística (Ondarza, 1911) donde accedimos a la versión completa que citamos en varias ocasiones. Se trata de un documento que se encontraba en el archivo personal de Manuel Vicente Ballivián. Los editores del Boletín mencionan: «su grande importancia será reconocida por todos aquellos que se interesen en conocer la historia cartográfica de la Republica» (Ondarza, 1911: 596).

20 El énfasis es nuestro. No hay una correlación clara de los años anotados en el «Autógrafo». Ondarza mencionó que la Comisión Topográfica fue creada en 1842, pero la carta de Bertrés enviada al ministro de Guerra por la que aceptó elaborar el mapa general de Bolivia fue fechada del 19 de agosto de ese año. Como mencionamos antes, la formalización de la creación de la «Mesa Topográfica» sería al año siguiente, el 8 de julio de 1843. De igual modo, Ondarza nos hace suponer que la Comisión Estadística fue creada el año de finalización del «Mapa Corográfico», 1843, pero esta sería creada por Ballivián dos años más tarde, en 1845. Estos cambios de fechas pueden haber sido intencionales por parte de Ondarza, a fin de justificar y reforzar sus argumentos ante distintos gobiernos mientras realizaba las gestiones para recuperar el dinero que se le debía por la impresión de los mapas de Bolivia, como detalla Mariano Baptista Gumucio en su artículo «El mapa que inició la deuda externa en Bolivia» (1988). 
atención que, si el mapa elaborado por Bertrés iba a ser utilizado por los miembros de la Comisión Estadística, en ninguna parte del Bosquejo Estadístico de Bolivia de José María Dalence (1851) o en los documentos sobre esta Comisión conservados en el Archivo y Biblioteca Nacionales de Bolivia, aparece una mención al «Mapa Corográfico».

\section{SEGUNDA FASE: AFIANZAMIENTO DE LA COMISIÓN Y PROYECCIÓN DEL SEGUNDO MAPA OFICIAL DE BOLIVIA}

Tras culminar su mapa general del país, en una carta de septiembre de 1843 Bertrés sugirió al ministro de Guerra una visita a cuatro regiones siguiendo rutas específicas. Más allá de tratarse de espacios que no fueron visitados por el ingeniero francés, eran una propuesta a fin de que sean estudiados y descritos de modo científico,

... teniendo en concideracion los grandes progresos que debe prometerse la Nacion por el descubrimiento de nuevas rutas cuyo trancito sea mas corto, ó mejora de las que hoy tenemos, que sin duda deberá formarse al levantar dichas topografías é Itinerarios: rutas por las que no solamente se puede esperar una facilitación respecto al comercio y para los transportes que un simple ciudadano puede hacer evitándose los fastos y penurias consiguientes á un largo viage, de nuevos moradores precisos á semejantes tráncitos. Estas reflecciones me han determinado suplicar á V.G. se digne de elevar al conocimiento de S.E. la presente nota á fin de que se sirva disponer lo que á este respecto crea mas acertado21.

La carta está acompañada por una «relación» de cuatro caminos o «[...] lugares que se deben recorrer y formar sus topografías é Itinerario acompañándose la Estadística correspondiente»: el camino desde Oruro hasta el Puerto de la Mar y el regreso por Potosí; desde Cochabamba hasta el embarcadero del río La Paz en la región de Yungas; desde Cochabamba hasta la confluencia con el río Mamoré, a fin de beneficiar al departamento de Beni creado cerca de un año atrás; y, finalmente, desde Tarija hasta el puerto de Magariños por el río Pilcomayo y de regreso hasta llegar a la confluencia de los ríos Pilaya y Pilcomayo22. Su finalidad era proyectarlos para que, a partir de ellos, se facilite el comercio y las comunicaciones y se mejoren los transportes, la agricultura y la ganadería, entre otros. Ballivián pudo tomar en cuenta las proposiciones de Bertrés y marcó así una segunda etapa de la comisión con la exploración in situ del territorio por parte de los miembros de la Comisión Topográfica, necesaria para lograr la precisión que Bertrés no había conseguido con el «Mapa Corográfico».

21 ABNB, MG, 20b, Carta del Departamento de Ingenieros al ministro de Guerra. Sucre, 23 de septiembre de 1843. El énfasis es nuestro.

22 ABNB, MG, 20b, Relación de los lugares que se deben recorrer y formar sus topografías é Itinerario acompañándose la Estadistica correspondiente. Sucre, 23 de septiembre de 1843. 
Acorde con esto, a mediados de 1844 el gobierno mandó formar dos subcomisiones: la primera, compuesta por Ladislao Marín, Manuel F. Escobar y Luis Camacho23, debía levantar la carta topográfica del departamento de Beni, mientras la segunda, integrada por Juan Ondarza y Juan Mariano Mujía, se encargaría del resto del país, comenzando su labor por el departamento de Cochabamba (Ondarza, 1911: 597). Hasta el mes de octubre, la «Sección de Topografía» funcionó en Sucre. A partir del mes de noviembre de 1844 sus actividades fueron registradas en el departamento de Cochabamba24, probablemente con Ondarza a la cabeza. Este hecho coincide con el retorno de Bertrés a Argentina25.

En una carta sin destinatario identificado fechada del 9 de abril de 1845, el comandante Ladislao Marín y los adjuntos, el subteniente Lucio Camacho y el Dr. Salvador Escobar, informaron haber recorrido en menos de un año «la vasta estension del territorio Beniano y navegado todos sus ríos» detallando su situación geográfica y demarcando los límites interiores y exteriores del departamento de Beni26. Lo llamativo de esta exploración — que se escribe en la carta- es la existencia de un cuadro, un diario de viaje y una colección de historia natural (pájaros, animales cuadrúpedos, insectos, mariposas), cuyo paradero desconocemos. En su memoria a las cámaras constitucionales de 1846, el ministro de Guerra Josef María Silva hizo referencia a esta colección:

Sin limitarse á sus funciones la comisión enviada al Beni, ha reunido una colección de áves preciosas de distintas clases, varios cuadrúpedos, insectos i otras curiosidades correspondientes al ramo de la historia natural (Silva, 1846: 5)27.

Más adelante, en una carta firmada por Ladislao Marín y dirigida a José Ballivián, se indica haber recibido la orden de elaborar el plano topográfico del departamento de Beni. Junto a esto, Marín también sugirió que se hiciesen las diligencias necesarias para subir y navegar los ríos Guaporé (o Iténez) y Verde (en los departamentos de Santa Cruz y Beni)28 que, al parecer, la subcomisión había dejado pendientes. No encontramos mayores detalles de la subcomisión formada por Marín, Escobar y Camacho; Ondarza señaló que esta no habría entregado ningún tipo de descripción o informe, pero eso ya no era necesario porque él y Mujía contaban con la información suficiente para cartografiar esa parte del

23 «Luis Camacho», como aparece en el documento «Autógrafo», es seguramente Lucio Camacho, quien se incorporó de manera formal a la Comisión Topográfica en 1846.

24 ABNB, MG, 1844, 4. Presupuesto General del haber mensual del Ejército, Comandancias Generales de los departamentos y demás establecimientos de la República en el mes de noviembre. La Paz, 30 de noviembre de 1844 .

25 La fecha de la última comunicación de Bertrés con el ministro de Guerra que encontramos en el Archivo y Biblioteca Nacionales de Bolivia fue del 5 de junio de 1844 en Yotala.

${ }_{26}$ ABNB, ARC-P82. Carta de la Comisión Topográfica del departamento de Beni./, 9 de abril de 1845.

27 Este aspecto es descrito también por Carmen Beatriz Loza en su artículo «François Rossignon, un naturalista francés cautivo de las aves del Caupolicán (Beni y La Paz, 1833-1847)» que brinda un papel protagónico a Ladislao Marín (Loza, 2005: 72-76), miembro de una de las subcomisiones.

28 ABNB, ARC-C464. Carta del Comandante D. Ladislao Marín a José Ballivián. «Commando Militar do Forthe do Principe da Beira», 27 de noviembre de 1845. El documento se encuentra escrito 
territorio29. La subcomisión comandada por Marín había enviado su mapa al Ministerio de Guerra, pero nunca llegó a manos de los jefes de la Comisión, Juan Ondarza y Juan Mariano Mujía.

El trabajo de los cartógrafos también se sometió a condiciones adversas. Ondarza y Mujía se fueron formando en la Mesa Topográfica de Bertrés, anexa al Estado Mayor General, entre 1843 y 1844. Para este último año habían iniciado su trabajo en el departamento de Cochabamba, pero todavía realizaban levantamientos preliminares. Cabe mencionar que en 1844 Ondarza tenía diecisiete años, si tomamos en cuenta que había luchado en la batalla de Ingavi (1841) tan solo con catorce años de edad. A pesar de estos «ensayos» de trabajo, se contaba con la venia del Gobierno que confiaba en el proyecto.

Pero el trabajo de la Comisión duró el tiempo que Ballivián fue presidente. A su salida, y ya con Belzu como mandatario, se detuvieron las exploraciones por el país bajo tuición estatal; su fase final no pudo concretarse por la oposición política existente entre este y aquel. Si es como indica Ondarza, después de 1847 el levantamiento topográfico de datos faltantes corrió por cuenta de él y de Mujía, pero no en calidad de comisionados. Ello se verifica igualmente en las fuentes existentes sobre el tema después de 1847; no encontramos, por ejemplo, documentación que trate acerca de los trabajos de la Comisión desde 1848, el año posterior al que Ballivián dejara el poder, hasta 185830.

La dispersión de las operaciones topográficas también afectó. Tomando un ejemplo, la Comisión Corográfica de Colombia tuvo fases definidas por los viajes de exploración a provincias específicas de aquel país entre 1850 a 1859, año en el cual su trabajo se detuvo por el fallecimiento del jefe de la Comisión31. Todos los viajes en las diez rutas transitadas partían de la capital colombiana, Bogotá, y volvían al mismo punto (Del Castillo, 2018: 148); el sitio era estratégico ya que allí se encontraban el Observatorio Astronómico y el Colegio Militar (Del Castillo, 2018: 148). En la Comisión Topográfica de Bolivia esa proyección desde una única ciudad matriz no existió. El plan de recolección de información contemplaba la visita a diferentes departamentos, cada uno con sus capitales que se convertían en el centro de operaciones. Si bien la Comisión Topográfica debía funcionar

en portugués de la época y está dirigido al «llustrisimo Señon», que presumimos se trata de José Ballivián.

${ }^{29}$ En su documento «Autógrafo», Ondarza mencionó que «jamás ha llegado á nuestras manos el Mapa del Beni recomendado á una comisión especial», pero que «no nos era necesario, habiendo recorrido nosotros mismos cuasi toda su extencion levantado las cartas de Caupolicán, Yuracarés, y determinado el curso de los Ríos que riegan aquel territorio» (Ondarza, 1911: 598).

30 En el ABNB solamente existen registros de los miembros de la Comisión Topográfica, y como «Departamento Topográfico», entre los años 1842 y 1847, con documentos que describen partidas de sueldos de Juan Ondarza, Juan Mariano Mujía, Lucio Camacho y sus criados. Para el año 1853 aparece un registro de la Comisión Topográfica en el catálogo del Fondo del Ministerio de Guerra,/; sin embargo, al solicitarlo no fue encontrado por los funcionarios del mencionado repositorio.

31 La Comisión Corográfica colombiana cuenta con una amplia bibliografía. Uno de los más completos y últimos trabajos es el de Nancy Appelbaum, Dibujar la nación. La Comisión Corográfica en la Colombia del siglo XIX (2017). 
desde la ciudad de Sucre y luego La Paz, debido a la extensión del territorio debió tener varios epicentros. En cada ciudad, sus miembros debían integrarse al ejército por su calidad de comisionados, pero sobre todo por temas financieros. También demostraba el enorme obstáculo que significaban la amplitud del terreno y las precarias vías de comunicación, que alejaban la posibilidad de controlar el territorio boliviano y aletargaban su mapeo.

Cuadro 1 - Algunas comisiones de topografía y geología a mediados del siglo XIX ${ }^{32}$

\begin{tabular}{|c|c|c|}
\hline $\begin{array}{c}\text { Años de duración/ } \\
\text { creación }\end{array}$ & País o PROVINCIA & Nombre \\
\hline 1824 & $\begin{array}{c}\text { Buenos Aires } \\
\text { (Argentina) }\end{array}$ & Comisión Topográfica \\
\hline 1826 & Argentina & $\begin{array}{c}\text { Departamento General de Topografía y } \\
\text { Estadística } \\
\text { (que reemplazó a la Comisión de 1824) }\end{array}$ \\
\hline 1843-1847 & Bolivia & Comisión Topográfica \\
\hline 1849-1859 & España & Comisión de la Carta Geológica \\
\hline 1850-1859 & Colombia & Comisión Corográfica \\
\hline 1852 & Argentina & $\begin{array}{c}\text { Departamento Topográfico (reorganizado } \\
\text { y que reemplazó a los dos anteriores de } \\
1824 \text { y 1826) }\end{array}$ \\
\hline 1853-1859 & España & Comisión de la Carta Geográfica \\
\hline 1862 [1849] & Entre Ríos (Argentina) & Departamento Topográfico de Entre Ríos \\
\hline 1865-1879 & Argentina & Mesa de Ingenieros (oficina militar) \\
\hline
\end{tabular}

Fuente: Elaboración propia en base a Arístegui et al. (2015); Bressan (2017); D’Angostino (2012); Del Castillo (2018); Díaz, Muñoz y Nieto (2010); Pesoa (2017) y Mazzitelli (2018)

Con ello se suspendieron además la articulación de los mapas topográficos departamentales en el mapa general y su posterior publicación. Recién a finales

32 Una lectura a los años en el cuadro ayuda a comprender la voluntad científica de los gobiernos por los estudios topográficos y geológicos en el siglo XIX. Para darle más utilidad, queda pendiente a futuro un estudio comparativo de las diferencias, similitudes y relaciones entre distintas comisiones de ese siglo en la región. 
de 1857, en una coyuntura más favorable, Ondarza y Mujía hicieron una solicitud al gobierno de José María Linares33, afín a Ballivián, para litografiar el mapa geográfico que habían elaborado desde la década anterior y para que su publicación fuera auspiciada por el Estado (fig. 2). Con el incentivo del ministro de Instrucción Pública, Lucas Mendoza de la Tapia, la solicitud fue discutida y aprobada finalmente por las autoridades mediante Instrucción Pública de fecha 8 de marzo de 1858 (Gaceta del Gobierno, 12.VI.1858).

De esta manera, el gobierno asumió los gastos de edición y litografía del mapa. Asimismo, se regularía su internación en el país y su uso como elemento dedicado a la enseñanza en los establecimientos del Estado cumpliendo, ante todo, una función educativa (ser utilizado para la instrucción primaria, la educación superior y castrense) 34 .

El gobierno selló la institucionalización de la cartografía con la creación de la Comisión Topográfica, la misma que elaboró dos instrumentos resultados de un proceso cartográfico en relación con el conocimiento de territorio. Pero este proceso de institucionalización de la cartografía boliviana fue, podríamos decirlo, breve. Como describimos, inició con el encargo de elaborar un mapa, el de 1843. Posteriormente trató de apoyarse en el proyecto de la Mesa Topográfica, se fortaleció con el trabajo de la Comisión Topográfica y finalizó con la publicación del mapa en 1859. No tenemos mayor dato de una comisión tal después de este año hasta finales del siglo XIX y principios del siglo XX, cuando surgirá ese llamado «periodo geográfico» que estuvo caracterizado por la creación de instancias gubernamentales como la Oficina Nacional de Estadística y Propaganda Geográfica que, encabezada por el geógrafo y estadista Manuel Vicente Ballivián, impulsó la escritura y reedición de varias obras geográficas coloniales y republicanas. Es así que la Comisión Topográfica sentó las bases de un «periodo geográfico» previo, caracterizado por las primeras exploraciones con carácter científico enviadas por el gobierno. En el caso específico, estas acciones determinaron los inicios de la cartografía estatal boliviana.

33 Linares nació en Ticala (intendencia de Potosí) en 1808. Ejerció varios cargos políticos para el Estado y ocupó la silla presidencial de Bolivia entre 1857 y 1861, cuando fue derrocado. Se exilió en Chile donde murió a los pocos meses (Barnadas, T. II, 2002: 80-81).

34 No podemos dejar de mencionar la deuda que había acumulado Bolivia con J. H. Colton, el impresor de los mapas, como expresaron Ondarza y Mujía en su folleto Recurso ante la Soberana Asamblea Constituyente de 1861. Estos indicaron que, una vez identificados los lugares de impresión, se eligió la mejor opción que era la propuesta por el Sr. J. H. Colton, «Jefe de la más respetable oficina de publicaciones geográficas en toda la Unión Americana» (Ondarza \& Mujía, 1861: 2), y así se firmó el contrato por la impresión de 10000 ejemplares de los mapas de Bolivia. Habiendo recibido nada más que el adelanto de los 2000 pesos que le fueron entregados en Nueva York, Colton cumplió con las fechas de entrega y los mapas Ilegaron al puerto peruano de Arica en 1860 en más de 400 cajones, junto con libros e instrumentos de instrucción pública encomendados por el gobierno de José María Linares, pero aún se le adeudaba 25500 pesos. El tema se dilató y quedó reflejado en el libro Oddments of Andean Diplomacy and other Oddments de Hinton Rowan Helper (1879), ubicado por Mariano Baptista Gumucio en la Biblioteca del Congreso en Washington (Baptista Gumucio, 1988: 98). Se trata de una compilación de la correspondencia enviada y recibida por el abogado de Colton, el mismo Helper, para resolver el asunto de la deuda contraída por Bolivia. 


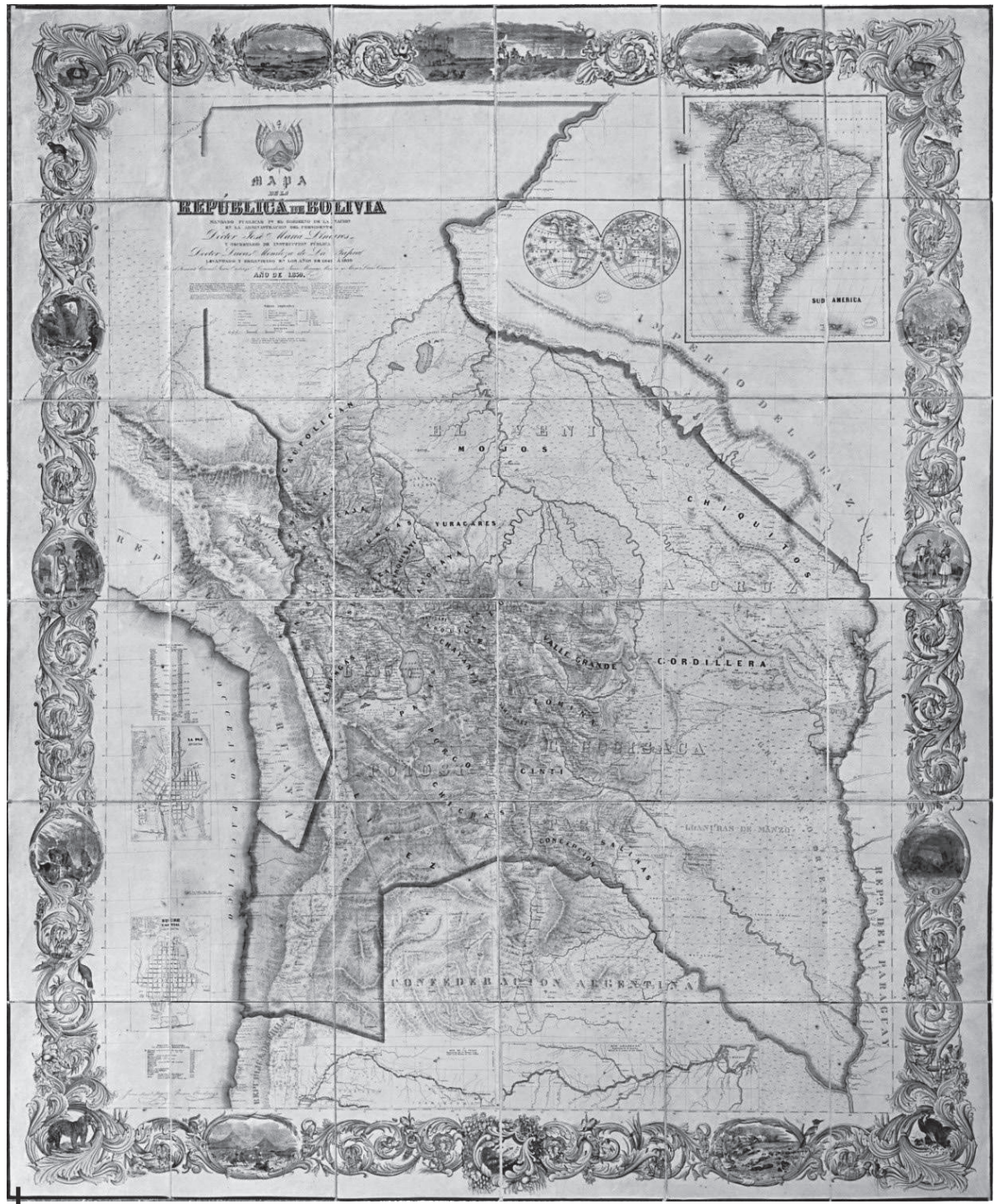

Figura 2 - Mapa de la República de Bolivia (1859)

Fuente: Grabado, impreso y publicado por J. H. Colton, Nueva York, 1859. http://catalogo.bn.gov. ar/F/?func $=$ direct\&doc_number $=000833242 \&$ local_base $=$ GENER Biblioteca Nacional de Argentina Mariano Moreno (Buenos Aires)

\section{CONCLUSIÓN}

El encargo del gobierno de José Ballivián a Felipe Bertrés para la elaboración de una carta geográfica del país en agosto de 1842 y la creación de la Mesa Topográfica en julio de 1843, fueron el inicio de un proyecto que repercutió en 
la publicación de los dos primeros mapas oficiales de Bolivia. Recapitulamos que Bertrés, siendo encargado de dicha Mesa, llevó adelante la elaboración del primer mapa en poco más de un año. En una segunda fase, Juan Ondarza y Juan Mariano Mujía, ya como parte de la Comisión Topográfica, siguieron los pasos de Bertrés y, a la postre, mejoraron su trabajo, aunque por avatares publicarían su mapa recién en 1859. Así, los dos primeros mapas oficiales bolivianos iniciaron por motivación de Ballivián y como producto de una institución creada por él.

José Ballivián, Felipe Bertrés y Juan Ondarza fueron los protagonistas que aportaron al conocimiento de nuestra geografía a mediados de aquella centuria. Su actuación, como gobernante uno y cartógrafos los otros, tuvo influencia en la consciencia actual del territorio boliviano, cuya dimensión se concretó en dos mapas oficiales, los únicos en el siglo XIX. Ambos fueron parte de un mismo proyecto que pretendía plasmar el territorio nacional en una representación oficial, consolidando de este modo la institucionalización de la ciencia geográfica y la cartografía de Estado. Pero a pesar de los esfuerzos, la Comisión Topográfica solamente tuvo vigencia durante del gobierno de José Ballivián.

En la investigación abordamos elementos referidos a la creación y la conformación de la Comisión Topográfica como tal; no estudiamos detalles de los mapas en sí. Consideramos dejar para un siguiente trabajo de investigación aspectos más puntuales de los mapas topográficos que resultaron de la Comisión Topográfica y la exploración de sus miembros por el país a mediados del siglo XIX, además de estudios pormenorizados de los mapas presentados.

\section{Referencias citadas}

\section{Hemerografía}

La Gaceta del Gobierno (1842, 1858)

Fondos documentales

Archivo y Biblioteca Nacionales de Bolivia, Sucre (ABNB)

Fondo: Ministerio de Guerra (MG)

Fondo: Archivo Raro y Curioso (ARC)

Biblioteca y Archivo Histórico de la Asamblea Legislativa Plurinacional, La Paz (BAH-ALP)

Fondo: Colección Oficial de Leyes, Decretos, Órdenes y Resoluciones Supremas

\section{Bibliografía}

\section{Fuentes primarias publicadas}

ARANZÁES, N., 1915 - Diccionario Histórico del Departamento de La Paz, 813 pp.; La Paz: Casa Editora Talleres Gráficos «La Prensa».

BALLIVIÁN, J., 1843 - Mensaje del Presidente de Bolivia a la Convención Nacional reunida en 184318 pp.; La Paz: Imprenta de Beeche y Cía.

BALLIVIÁN, M. V. \& IDIÁQUEZ, E., 1890 - Diccionario Geográfico de la República de Bolivia. Tomo Primero: Departamento de La Paz, XV + 164 pp.; La Paz: Imprenta y Litografía de «El Nacional» de Isaac V. Vila. 
DALENCE, J. M., 1851 - Bosquejo Estadístico de Bolivia, 391 pp.; Chuquisaca: Imprenta de Sucre.

DÍAZ ANGEL, S., MUÑOZ ARBELÁEZ, S. \& NIETO OLARTE, M., 2010 - Ensamblando la nación: cartografía y política en la historia de Colombia, 103 pp.; Bogotá: Ediciones Uniandes, Facultad de Artes y Humanidades, Facultad de Ciencias Sociales, Departamento de Historia, Centro de Estudios Socioculturales e InternacionesCESO.

HELPER, H. R., 1879 - Oddments of Andean Diplomacy and other Oddments, 480 pp.; St. Louis, Missouri: Ryan, Jacks \& Co. Printers.

ONDARZA, J., 1911 - Autógrafo del señor Ondarza. Boletín de la Oficina Nacional de Estadística. 1911, 64/65/66: 596-600; La Paz: Tip. Comercial de Ismael Argote, Editor.

ONDARZA, J. \& MUJÍA, J. M., 1861 - Recurso ante la Soberana Asamblea Constituyente de 1861, 8 pp.; La Paz: Imprenta Paceña.

SILVA, J. M., 1846 - Memoria del Ministro de la Guerra a las Cámaras Constitucionales de 18466 рp.; Sucre: Imprenta de Beeche y Cía.

\section{Fuentes secundarias}

APPELBAUM, N., 2017 - Dibujar la nación. La Comisión Corográfica en la Colombia del siglo XIX, 360 pp.; Bogotá: Universidad de los Andes-Ediciones Uniandes, Fondo de Cultura Económica.

ARÍSTEGUI CORTIJO, A., RUÍZ RAMÍREZ, A. d. C. \& DÁVILA MARTíNEZ, F. J., 2015 - Las Comisiones del Mapa de España en la década de 1850. Anales de Geografía, 35 (2): 9-44.

BAPTISTA GUMUCIO, M., 1988 - El mapa que inició la deuda externa de Bolivia. Historia y Cultura, 14: 97-106; La Paz: Sociedad Boliviana de la Historia, Editorial Don Bosco.

BARNADAS, J., 2002 - Diccionario Histórico de Bolivia, 2 vols., 1152 pp.; Sucre: Grupo de Estudios Históricos.

BRESSAN, R. V., 2017 - Registrar, ordenar y planificar. El Departamento Topográfico de Entre Ríos, 1871-1883. Revista Estudios Sociales Contemporáneos, 17: 36-52.

BARRAGÁN ROMANO, R., LEMA GARRETT A. M., MENDIETA PARADA, P., PERES-CAJÍAS, J. \& URCULLO PEREIRA, A., 2015 - Bolivia, Su Historia. Tomo IV: Los primeros cien años de la Republica 1825-1925, 375 pp.; La Paz: Coordinadora de Historia.

COSTA DE LA TORRE, A., 1970 - Hombres célebres de Bolivia, Juan Ondarza, autor del mapa de Bolivia; Abdón Senen Ondarza, fundador de Antofagasta, 370 pp.; La Paz: Imprenta y Librería «Renovación» Ltda.

D'ANGOSTINO, V. A., 2012 - Estado, instituciones y funcionarios: el Departamento Topográfico bonaerense en la segunda mitad del siglo XIX. In: III Congreso Latinoamericano de Historia Económica y XXIII Jornadas de Historia Económica (A. Regalsky, Presidencia); San Carlos de Bariloche.

DEL CASTILLO, L., 2018 - La invención republicana del legado colonial. Ciencia, historia y geografía de la vanguardia política colombiana en el siglo XIX, 360 pp.; Bogotá: Ediciones Uniandes, Banco de la República de Colombia.

DÍAZ ARGUEDAS, J., 1929 - Los generales de Bolivia (rasgos biográficos), 1825-1925, 718 pp.; La Paz: Imp. Intendencia Gral. de Guerra.

GARCÍA JORDÁN, P., 2001 - Cruz y arado, fusiles y discursos. La construcción de los Orientes en el Perú y Bolivia, 1820-1940, 476 pp.; Lima: IFEA, IEP. 
GROFF GREEVER, J., 1987 - José Ballivián y el oriente boliviano, xvi + 224 pp.; La Paz: Empresa Editora Siglo Ltda.

LAFUENTE, A. \& LÓPEZ-OCÓN, L., 1998 - Bosquejos de la ciencia nacional en la América Latina del siglo XIX: Introducción. Asclepio, 50 (2): 5-10.

LOZA, C. B., 2005 - François Rossignon, un naturalista francés cautivo de las aves del Caupolicán (Beni y La Paz, 1833-1847). Bulletin de I'Institut francais d'études andines, 34 (1): 59-80.

MAZZITELLI MASTRICCHIO, M., 2018 - Los «Jueces de la Medida», el rol de los agrimensores entrerrianos en el Departamento Topográfico de Entre Ríos (18491871). Revista Brasileira de História da Ciência, 1: 7-20.

PESOA MARCILLA, M., 2017 - Conocer, medir y dibujar el territorio. Orígenes y etapa formativa del Departamento Topográfico de Buenos Aires (1824-1851). Boletín Americanista, 75: 153-172.

SALDAÑA, J. L., 2006 - The Latin American Scientific Theater. In: Science in Latin America A history (J. L. Saldaña, ed.): 1-27; Austin: University of Texas Press.

SALINAS, J. M., 1967 - Historia de la Universidad Mayor de San Andrés, Tomo Primero, 652 pp.; La Paz: UMSA.

YABEN, J., 1938 - Biografías argentinas y sudamericanas, 5 vols.; Buenos Aires: Editorial Metropolis. 